\title{
PELLE DI TILAPIA: PROGRESSO TECNOLOGICO NEL TRATTAMENTO DELLE USTIONI?
}

\section{REVISIONE ARTICOLO}

CABRAL, Anna Julie Medeiros ${ }^{1}$, LIMA, Camila Araújo Novais², CRUZ FILHO, Eduardo Franco Correia ${ }^{3}$, SOARES, Gabriel Lucena de Carvalho ${ }^{4}$, ESPÍNOLA, Paulo Francisco Lucena de Araújo ${ }^{5}$, ALVES, Fernanda Araújo6 ${ }^{6}$, CRUZ, Ana Suzy de Góis Melo ${ }^{7}$

CABRAL, Anna Julie Medeiros. Et al. Pelle di tilapia: progresso tecnologico nel trattamento delle ustioni?. Revista Científica Multidisciplinar Núcleo do Conhecimento. Anno. 06, Ed. 12, Vol. 05, pp. 50-64. Dicembre 2021. ISSN: 24480959, Link di accesso: https://www.nucleodoconhecimento.com.br/salute/pelle-ditilapia, DOI: 10.32749/nucleodoconhecimento.com.br/salute/pelle-di-tilapia

\section{RIEPILOGO}

Introduzione: La ustioni è una delle più grandi aggressioni che il corpo può subire. L'approccio varia in base al grado di ustione, perché l'uso di clorexidina, sulfadiazina d'argento,, sbrigliamento del tessuto necrotico, medicazioni biosintetiche e pelli artificiali. Tuttavia, questi ultimi due hanno costi elevati, quindi sono emersi diversi studi con l'obiettivo di cercare opzioni più praticabili, come l'uso della pelle di Tilapia del Nilo nelle ustioni, grazie alle sue proprietà curative. Detto questo, il presente articolo ha come guida la domanda: questo nuovo metodo, in effetti, è un progresso

\footnotetext{
${ }^{1}$ Studente del corso di Medicina del Centro Universitario di João Pessoa - UNIPÊ, João Pessoa - PB. ORCID: 0000-0002-5212-5521.

${ }^{2}$ Studente del corso di Medicina del Centro Universitario di João Pessoa - UNIPÊ, João Pessoa - PB. ORCID: 0000-0003-4609-4260.

${ }^{3}$ Studente del corso di Medicina del Centro Universitario di João Pessoa - UNIPÊ, João Pessoa - PB. ORCID: 0000-0001-6684-2161.

${ }^{4}$ Studente del corso di Medicina del Centro Universitario di João Pessoa - UNIPÊ, João Pessoa - PB. ORCID: 0000-0002-0618-4037

${ }^{5}$ Studente del corso di Medicina del Centro Universitario di João Pessoa - UNIPÊ, João Pessoa - PB. ORCID: 0000-0001-7964-2118.

${ }^{6}$ Consulente. ORCID: 0000-0001-5235-5020.

${ }^{7}$ Consulente. ORCID: 0000-0002-0668-0540.
}

RC: 104337

Disponibile in: https://www.nucleodoconhecimento.com.br/salute/pelle-di-tilapia 
tecnologico così importante per il trattamento dei pazienti ustionati come sembra essere? Obiettivo: Analizzare l'uso della pelle di Tilapia del Nilo in pazienti con ustioni, nonché confrontarla con altre tecniche prestabilite. Metodi: Si tratta di una rassegna bibliografica integrativa con un approccio qualitativo. I dati sono stati raccolti attraverso i database pubmed e la Biblioteca virtuale della salute (BVS), dal 2015 al 2020. Risultati: Gli articoli indicano una buona prognosi all'uso della pelle di Tilapia del Nilo in relazione alle altre opzioni in vigore per il trattamento delle ustioni, con un vantaggio significativo nel ridurre il numero di medicazioni richieste, a causa di una migliore stima della ferita. Inoltre, presenta caratteristiche microscopiche simili alla pelle umana, come l'elevata resistenza alla trazione e l'estensione della rottura, riducendo i tempi di epitelizzazione e l'intensità del dolore, oltre a ridurre i costi di trattamento. Conclusione: Alla luce dei risultati della letteratura riportati nella presente revisione, si conclude che gli studi con pelle di tilapia del Nilo si rivelano una modalità rivoluzionaria con benefici nel trattamento di pazienti con lesioni cutanee superficiali e profonde. Pertanto, i ricercatori hanno concluso, rispondendo alla domanda guida, che il nuovo metodo è, sì, un importante progresso nel campo del trattamento delle ustioni, perché la sua occupabilità è confermata, oltre a dimostrare un vantaggio rispetto ad alcune delle principali alternative preesistenti.

Parole chiave: Tilapia, Ustioni, Medicazioni Biologiche.

\section{INTRODUZIONE}

La velocità e lo stile di vita imposti dalla modernità fanno aumentare oggi l'incidenza del numero di ustioni. Ben oltre le lesioni fisiche, le ustioni sono anche responsabili di problemi economici associati a danni irreparabili ai pazienti e alle loro famiglie (HU et al., 2017). L'ustione è una delle più grandi aggressioni che l'organismo umano può subire, e si stima che in Brasile ci siano circa 1 milione di incidenti da ustione all'anno, secondo Leontsinis et al. (2018), sia che si tratti di un semplice colpo di calore, ustioni di primo grado, fino alla distruzione totale della pelle (epidermide e derma) e dei tessuti adiacenti, come nelle ustioni di terzo grado (ALVES et al., 2015). 
Quando consideriamo il trattamento delle ustioni in Brasile, ci troviamo di fronte a due realtà: quella della rete pubblica e privata. Nei centri di trattamento della rete pubblica, la condotta seguita in relazione alle medicazioni in lesioni di secondo grado è il bagno giornaliero con clorexidina al $2 \%$ e la medicazione viene eseguita con l'antimicrobico topico argento sulfadiazina all'1\% fino alla completa riparazione della lesione; mentre nelle ustioni di terzo grado, il tessuto necrotico viene sbrigliato in più fasi, quindi la medicazione con sulfadiazina d'argento all'1\% viene eseguita fino a quando il letto della ferita non viene preparato per l'innesto. D'altra parte, nella rete privata, tenendo conto delle diverse condizioni finanziarie di ciascun paziente, medicazioni biosintetiche e pelli artificiali, possono essere utilizzate opzioni importate e ad alto costo (ALVES et al., 2015).

Tuttavia, analizzando la realtà vissuta in Europa e negli Stati Uniti, il trattamento prevede spesso l'uso di pelli omologhe (facendo uso di banche cutanee), eterologo (di origine animale), medicazioni biosintetiche e derma artificiale (ALVES et al., 2015). In Brasile, ci sono ancora alcuni scontri per ottenere la stessa praticità di questi altri paesi. Culturalmente, c'è ancora una grande resistenza alla donazione della pelle, che finisce per limitare la disponibilità del tessuto per soddisfare la grande domanda. Inoltre, prendendo come riferimento il Ministero della Salute, in modo che il Brasile possa soddisfare la necessità di pelle omologo per l'uso in ustioni, sarebbe necessario avere 13 banche della pelle distribuite su tutto il territorio nazionale, tuttavia ce ne sono solo cinque (San Paolo, Curitiba, Rio de Janeiro, Porto Alegre e Recife - che è disabile). Oltre a queste limitazioni, il Brasile non ha mai avuto una pelle di animale registrata presso l'Agenzia nazionale di sorveglianza sanitaria (ANVISA) e resa disponibile dal Sistema sanitario unificato (SUS), che finisce anche per limitare l'uso della pelle eterologo nei pazienti ustionati (LEONTSINIS et al., 2018).

Pertanto, sono emersi diversi studi con l'obiettivo di cercare medicazioni da utilizzare nei pazienti ustionati. Queste medicazioni mirano a ridurre gli effetti della contaminazione sulle lesioni, favorire il processo di guarigione e offrire migliori risultati estetici. Avere, idealmente, buona flessibilità e aderenza al letto, resistenza 
allo stretching, maneggevolezza, capacità di sopprimere il dolore, basso costo e facile da ottenere, oltre a prevenire perdite idroelettrolitiche, contaminazione batterica, favorisce la riepitelizzazione delle ustioni e fornisce la formazione di un adeguato tessuto di granulazione.Una delle opzioni è la ricerca di sostituti temporanei della pelle e medicazioni di materiali sintetici o biosintetici, perché riducono la frequenza del cambio di medicazione, tuttavia, gli alti costi e la sua inefficacia per le ustioni profonde hanno stimolato la ricerca di materiali biologici come alternative praticabili (COSTA et al., 2019; MARCELO e BRANDT, 2019).

In questo contesto, nel 2011, il chirurgo plastico di Pernambuco Marcelo Borges, dopo aver visto un articolo su Jornal do Commercio de Pernambuco che parlava dell'uso della pelle della specie Tilapia del Nilo (Oreochromis niloticus) nell'artigianato, uno dei pesci più coltivati in Brasile, per primo è venuta l'idea di utilizzare questa pelle nel trattamento delle ustioni. Ma è stato solo nel 2014 che ha condiviso l'idea con Edmar Maciel, chirurgo plastico del Ceará, iniziando studi sull'uso della pelle di Tilapia del Nilo nelle ustioni. Anche il ricercatore Odorico Moraes, direttore presidente del Centro di ricerca e sviluppo della medicina (NPDM), presso l'Università federale del Ceará (UFC), e il chirurgo plastico Nelson Piccolo di Goiás sono stati integrati per coordinare il lavoro (LIMA JUNIOR et al., 2017).

Come narrato da Lima Junior (2017), la pelle di Tilapia del Nilo mostra un'epidermide rivestita da un epitelio pavimentale stratificato, seguito da ampi strati di collagene. Grazie alla sua caratteristica di guidare e definire la maggior parte dei tessuti, si configura come un grande componente dei biomateriali, oltre a consentire biodegradabilità e biocompatibilità, che ne favoriscono l'applicazione (LIMA JUNIOR, 2017; LIMA JUNIOR et al., 2019b, 2020b).

Detto questo, la domanda guida è: "Questo nuovo metodo, infatti, è un progresso tecnologico così importante per il trattamento dei pazienti ustionati come sembra?" L'obiettivo, quindi, attraverso questo studio, è analizzare se la pelle di Tilapia del Nilo sia una valida alternativa da utilizzare in pazienti con ustioni, nonché confrontarla con altre tecniche già utilizzate, verificando i vantaggi dell'adozione di questa nuova metodica. 
Più specificamente, questo studio mira a evidenziare il buon aforeth della medicazione della pelle tilapia nel trattamento delle ustioni, correlando le proprietà fisico-chimiche della tilapia con i benefici della medicazione ed esponendo la non inferiorità delle medicazioni cutanee tilapia rispetto ad altre soluzioni sul mercato. Oltre ad incentivare la realizzazione di ulteriori ricerche scientifiche per ottenere risultati più robusti sull'argomento.

\section{METODOLOGIA}

Si riferisce a una revisione della letteratura integrativa, con un approccio qualitativo, con scopo descrittivo di studi nazionali e internazionali. Inoltre, ha una natura applicata, con I'uso di una procedura bibliografica per l'analisi dei rispettivi dati raccolti relativi all'uso della pelle di tilapia in pazienti ustionati.

Il presente studio è stato condotto attraverso la ricerca di articoli attraverso l'accesso ai database di pubmed e alla Biblioteca virtuale della salute (BVS). In un primo momento, sono stati raccolti i descrittori rilevanti per il tema selezionato, essendo il vocabolario dei Descrittori in Scienze della Salute (DeCS). I termini scelti sono stati "Tilapia", "bruciare" e "Medicazioni biologiche" nelle lingue: portoghese, inglese e spagnolo. Questi descrittori sono stati associati agli operatori booleani "OR" e "AND" elaborando la seguente formula di ricerca: "(Tilápia OR Tilapia OR Tilapia) AND (Queimaduras OR Burns OR Quemaduras) AND (Curativos Biológicos OR Biological Dressings OR Apósitos Biológicos)".

Pertanto, applicando la logica della formula di cui sopra, sono stati trovati un totale di 31 articoli nelle banche dati selezionate, 14 articoli pubblicati e 17 disponibili nel BVS. I criteri di inclusione erano: articoli disponibili per intero, che ritraevano il tema relativo alla revisione integrativa e articoli pubblicati e indicizzati nelle banche dati pubblicate negli anni 2015, 2016, 2017, 2018, 2019, 2020 e 2021, che presentavano la lingua in inglese, portoghese e spagnolo. Inoltre, gli articoli che sono stati duplicati, non avevano un contenuto completo e che non soddisfacevano gli obiettivi di ricerca sono stati esclusi dalla revisione integrativa. 
La revisione bibliografica integrativa non è pratica, pertanto non richiede la presentazione al Comitato etico della ricerca (CEP). In quest'ottica è stata elaborata una tabella che mostra le principali informazioni delle bibliografie utilizzate, presentando i dati relativi al titolo, agli autori, all'anno di pubblicazione, al paese e ai principali risultati di ciascuna.

\section{RISULTATI}

Tabella 1: Uso della pelle di tilapia nelle ustioni: basi teoriche e implicazioni pratiche.

\begin{tabular}{|c|c|c|c|}
\hline & titolo & $\begin{array}{l}\text { Autori, anno } \\
\text { di } \\
\text { pubblicazione } \\
\text { e paese }\end{array}$ & Principali risultati \\
\hline $\begin{array}{l}\text { ARTICOLO } \\
1\end{array}$ & $\begin{array}{l}\text { Uso da pele de tilápia } \\
\text { (Oreochromis niloticus), } \\
\text { como curativo biológico } \\
\text { oclusivo, no tratamento } \\
\text { de queimaduras }\end{array}$ & $\begin{array}{l}\text { Lima Júnior et } \\
\text { al., } 2017, \\
\text { Brasil }\end{array}$ & $\begin{array}{l}\text { Lo studio ha dimostrato } \\
\text { che la pelle di tilapia ha } \\
\text { una notevole aderenza } \\
\text { alle ferite da ustione nei } \\
\text { ratti di prova, con risultati } \\
\text { positivi durante la } \\
\text { guarigione, senza } \\
\text { mutazioni significative } \\
\text { negli scopi biochimici ed } \\
\text { ematologici della } \\
\text { funzionalità epatica e } \\
\text { renale, costituendo una } \\
\text { possibilità di medicazione } \\
\text { biologica. }\end{array}$ \\
\hline $\begin{array}{l}\text { ARTICOLO } \\
2\end{array}$ & $\begin{array}{l}\text { Xenoenxerto (pele da } \\
\text { Tilápia-do-Nilo) } \\
\text { hidrofibra com prata no } \\
\text { tratamento }\end{array}$ & $\begin{array}{l}\text { De Miranda e } \\
\text { Brandt, 2019, } \\
\text { Brasil }\end{array}$ & $\begin{array}{l}\text { La ricerca ha dimostrato } \\
\text { che Tilapia del Nilo è } \\
\text { efficace nella funzione di } \\
\text { medicazione biologica }\end{array}$ \\
\hline
\end{tabular}


queimaduras de II grau

em adultos

occlusiva nel trattamento

delle ustioni di secondo

grado negli adulti, con

mezzi simili per quanto

riguarda il tempo di

trattamento, il riferimento

al dolore e la necessità di sostituzione, per quanto riguarda la gestione dei pazienti con Aquacel $A G \AA$.

ARTICOLO Avaliação microscópica, Alves et al., La pelle di Tilapia 3 estudo histoquímico e 2015, Brasil análise de propriedades tensiométricas da pele de tilápia do Nilo presenta fasci di fibre di collagene dense, prevalentemente di tipo 1, che presentano caratteristiche microscopiche simili a quelle della pelle umana, nonché un'elevata resistenza alla trazione e un'estensione alla rottura, rendendola promettente come biomateriale nella medicina rigenerativa.

\begin{tabular}{|l|l|l|l|lr} 
ARTICOLO & Innovative & Burn & Lima Júnior et & Lo studio clinico \\
& Treatment Using Tilapia & al., & 2020, & randomizzato ha \\
& Skin as a Xenograft: A & Brasil & utilizzato un campione di \\
& Phase II Randomized & & 62 partecipanti e ha \\
& Controlled trial & & dimostrato che c'era una \\
& & & riduzione di: il tempo di
\end{tabular}




\begin{tabular}{|c|c|c|c|}
\hline & & & $\begin{array}{l}\text { riepitelizzazione, } \\
\text { l'intensità del dolore, la } \\
\text { quantità di estetica } \\
\text { analgesici e la necessità } \\
\text { di cambiare medicazione } \\
\text { rispetto al gruppo di } \\
\text { controllo, quindi, la pelle } \\
\text { di tilapia presentava } \\
\text { benefici. }\end{array}$ \\
\hline $\begin{array}{l}\text { ARTICOLO } \\
5\end{array}$ & $\begin{array}{l}\text { Tratamento de } \\
\text { queimaduras de segundo } \\
\text { grau profundo em } \\
\text { abdômen, coxas e } \\
\text { genitália: uso da pele de } \\
\text { tilápia como um } \\
\text { xenoenxerto }\end{array}$ & $\begin{array}{l}\text { Lima Júnior et } \\
\text { al., } 2020, \\
\text { Brasil }\end{array}$ & 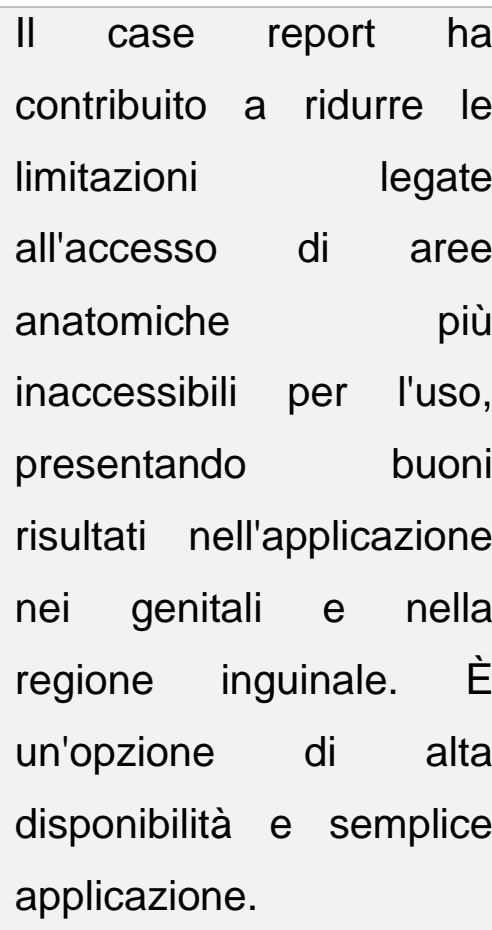 \\
\hline $\begin{array}{l}\text { ARTICOLO } \\
6\end{array}$ & $\begin{array}{l}\text { Marine Collagen Peptides } \\
\text { from the Skin of Nile } \\
\text { Tilapia (Oreochromis } \\
\text { niloticus): } \\
\text { Characterization and } \\
\text { Wound Healing } \\
\text { Evaluation }\end{array}$ & $\begin{array}{l}\text { Zhang } \mathrm{Hu} \text { et } \\
\text { al., } \\
\text { China }\end{array}$ & $\begin{array}{l}\text { Il test di raschiatura in } \\
\text { vitro e in vivo ha rivelato } \\
\text { conseguenze significative } \\
\text { nella chiusura della } \\
\text { raschiatura con pelle di } \\
\text { tilapia, con evoluzione nel } \\
\text { processo di guarigione } \\
\text { mediante scottature } \\
\text { profonde a spessore }\end{array}$ \\
\hline
\end{tabular}




\begin{tabular}{|c|c|c|c|}
\hline & & & $\begin{array}{l}\text { parziale nei conigli testati, } \\
\text { presentandosi come un } \\
\text { trattamento promettente. }\end{array}$ \\
\hline $\begin{array}{l}\text { ARTICOLO } \\
7\end{array}$ & $\begin{array}{l}\text { A Randomized } \\
\text { Comparison Study of } \\
\text { Lyophilized Nile Tilapia } \\
\text { Skin and Silver- } \\
\text { Impregnated Sodium } \\
\text { Carboxymethylcellulose } \\
\text { for the Treatment of } \\
\text { Superficial Partial- } \\
\text { Thickness Burns }\end{array}$ & $\begin{array}{l}\text { Lima Júnior, et } \\
\text { al., } 2020, \\
\text { Brasil }\end{array}$ & $\begin{array}{l}\text { Questo studio pilota ha } \\
\text { dimostrato che non vi è } \\
\text { alcuna inferiorità alla } \\
\text { pelle di tilapia liofilizzata } \\
\text { come opzione terapeutica } \\
\text { nelle ustioni, rispetto alla } \\
\text { medicazione sordida } \\
\text { carbossimetilcellulosa } \\
\text { impregnata d'argento, } \\
\text { con efficacia e sicurezza, } \\
\text { consentendo lo sviluppo } \\
\text { di studi clinici } \\
\text { randomizzati più } \\
\text { complessi in futuro. }\end{array}$ \\
\hline $\begin{array}{l}\text { ARTICOLO } \\
8\end{array}$ & $\begin{array}{lr}\text { Elaboração de } & \text { um } \\
\text { protocolo } & \text { para } \\
\text { implementação } & \text { e } \\
\text { funcionamento } & \text { do } \\
\text { primeiro banco de pele } \\
\text { animal do Brasil: Relato } \\
\text { de experiência }\end{array}$ & $\begin{array}{l}\text { Leontsinis et } \\
\text { al., 2018, } \\
\text { Brasil }\end{array}$ & $\begin{array}{l}\text { L'articolo ha rivelato } \\
\text { l'importanza di sviluppare } \\
\text { e implementare protocolli } \\
\text { per la prima banca di } \\
\text { pelle di animali acquatici } \\
\text { al mondo, rafforzando la } \\
\text { necessità di stabilire la } \\
\text { standardizzazione del } \\
\text { sistema. }\end{array}$ \\
\hline $\begin{array}{l}\text { ARTICOLO } \\
9\end{array}$ & $\begin{array}{l}\text { Use of Tilapia Skin as a } \\
\text { Xenograft for Pediatric } \\
\text { Burn Treatment: A Case } \\
\text { Report }\end{array}$ & $\begin{array}{l}\text { Costa et al., } \\
2019 \text {, Estados } \\
\text { Unidos }\end{array}$ & $\begin{array}{l}\text { Lo xenotrapianto cutaneo } \\
\text { di tilapia nel caso clinico } \\
\text { di una vittima di ustioni di } \\
3 \text { anni ha mostrato } \\
\text { risultati positivi con un }\end{array}$ \\
\hline
\end{tabular}




\begin{tabular}{|c|c|c|c|}
\hline & & & $\begin{array}{l}\text { significativo impatto } \\
\text { sociale e finanziario sul } \\
\text { sistema sanitario. }\end{array}$ \\
\hline $\begin{array}{l}\text { ARTICOLO } \\
10\end{array}$ & $\begin{array}{l}\text { Innovative treatment } \\
\text { using tilapia skin as a } \\
\text { xenograft for partial } \\
\text { thickness burns after a } \\
\text { gunpowder explosion }\end{array}$ & $\begin{array}{l}\text { Lima Júnior et } \\
\text { al., } 2019, \\
\text { Inglaterra }\end{array}$ & $\begin{array}{l}\text { Il caso clinico ritrae un } \\
\text { paziente maschio di } 23 \\
\text { anni con ustioni } \\
\text { superficiali e profonde } \\
\text { rispettivamente sull'arto } \\
\text { superiore destro e } \\
\text { sinistro. Con l'uso della } \\
\text { pelle di tilapia, la } \\
\text { riepitelizzazione è stata } \\
\text { eseguita in breve tempo, } \\
\text { senza effetti collaterali e } \\
\text { necessità di cambiare la } \\
\text { medicazione. }\end{array}$ \\
\hline $\begin{array}{l}\text { ARTICOLO } \\
11\end{array}$ & $\begin{array}{l}\text { Pediatric Burn Treatment } \\
\text { Using Tilapia Skin as a } \\
\text { Xenograft for Superficial } \\
\text { Partial-Thickness } \\
\text { Wounds: A Pilot Study }\end{array}$ & $\begin{array}{l}\text { Lima Júnior, } \\
2020, \\
\text { Inglaterra }\end{array}$ & $\begin{array}{l}\text { L'uso della pelle di tilapia } \\
\text { è stato utilizzato come } \\
\text { risorsa extra insieme alla } \\
\text { sulfadiazina d'argento. Lo } \\
\text { studio ha rivelato che } \\
\text { oltre a portare benefici ai } \\
\text { pazienti, ha ridotto i costi } \\
\text { di trattamento, così come } \\
\text { per gli operatori sanitari, } \\
\text { come la riduzione del } \\
\text { carico di lavoro. }\end{array}$ \\
\hline $\begin{array}{l}\text { ARTICOLO } \\
12\end{array}$ & $\begin{array}{l}\text { Comprehensive } \\
\text { Assessment of Nile } \\
\text { Tilapia Skin (Oreochromis } \\
\text { niloticus) Collagen }\end{array}$ & $\begin{array}{l}\text { Ge et al., } \\
2020 \text {, China }\end{array}$ & $\begin{array}{l}\text { La medicazione } \\
\text { allidrogel di collagene ha } \\
\text { mostrato la promozione } \\
\text { della formazione di strati }\end{array}$ \\
\hline
\end{tabular}




\begin{tabular}{|c|c|c|c|}
\hline & $\begin{array}{l}\text { Hydrogels for Wound } \\
\text { Dressings }\end{array}$ & & 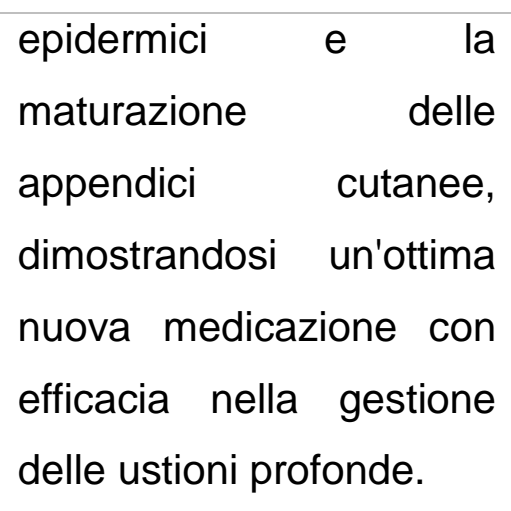 \\
\hline $\begin{array}{l}\text { ARTICOLO } \\
13\end{array}$ & $\begin{array}{l}\text { Tecnologias inovadoras: } \\
\text { uso da pele da tilápia do } \\
\text { Nilo no tratamento de } \\
\text { queimaduras e feridas }\end{array}$ & $\begin{array}{l}\text { Lima Júnior, } \\
\text { 2017, Brasil }\end{array}$ & $\begin{array}{l}\text { La pelle di Tilapia nelle } \\
\text { ustioni è un brevetto } \\
\text { brasiliano e l'obiettivo } \\
\text { della ricerca è la } \\
\text { registrazione della pelle } \\
\text { nell'Agenzia nazionale di } \\
\text { sorveglianza sanitaria } \\
\text { (ANVISA) e resa } \\
\text { disponibile dal Sistema } \\
\text { sanitario unificato (SUS). }\end{array}$ \\
\hline $\begin{array}{l}\text { ARTICOLO } \\
14\end{array}$ & $\begin{array}{l}\text { Chitosan hydrogel in } \\
\text { combination with marine } \\
\text { peptides from tilapia for } \\
\text { burns healing }\end{array}$ & $\begin{array}{l}\text { Ouyang et al., } \\
2018 \text {, China }\end{array}$ & $\begin{array}{l}\text { I peptidi marini estratti } \\
\text { dalla tilapia hanno } \\
\text { mostrato } \\
\text { composizione simile al } \\
\text { collagene, oltre a una } \\
\text { significativa attività } \\
\text { antibatterica. Presenta } \\
\text { un'elevata efficienza di } \\
\text { guarigione e capacità di } \\
\text { migrazione } \\
\text { proliferazione cellulare } \\
\text { rispetto al gruppo di } \\
\text { controllo e ad altri } \\
\text { unguenti disponibili }\end{array}$ \\
\hline
\end{tabular}


nell'industria

farmaceutica.

Fonte: Sviluppato dall'autore (2021)

La revisione ha incluso 14 articoli, tra i quali, due (14,3\%) hanno riportato i risultati utilizzando test sugli animali, il $14,3 \%$ su un'analisi comparativa tra trattamenti tradizionali e uso della pelle di tilapia e il $21,4 \%$ sui meccanismi di composizione e proprietà in relazione alla pelle umana. L'uso di studi randomizzati ha rappresentato il $7,1 \%$ degli articoli, il $28,6 \%$ ha presentato i risultati in case report e il $14,3 \%$ ha spiegato lo sviluppo della tecnica fino alla sua importanza nelle banche della pelle al giorno d'oggi.

Lo studio condotto presso il Centro per il trattamento delle ustioni dell'Istituto Dr. José Frota, Ospedale pubblico di Fortaleza, Ceará, Brasile, ha selezionato 62 pazienti ricoverati tra ottobre 2016 e settembre 2017, vittime di ustioni. Le varianti valutate sono state: il numero di volte in cui la medicazione occlusiva è stata cambiata e il numero di giorni per la guarigione della ferita, l'uso di analgesici o anestetici, la sensibilità al dolore, il miglioramento dell'ustione durante la rimozione della medicazione. Lo studio ha analizzato i dati e suddiviso i partecipanti, in base a: sesso, età, agenti responsabili dell'incidente, superficie corporea e segmenti ustionati, e per questo, suddiviso in tre classificazioni, A - pazienti ambulatoriali con ustioni a spessore parziale superficiale inferiore al 10\%, B - ricoverati con lo stesso dal 10 al 20\%, C - ricoverati con ustioni profonde a spessore parziale dal 5 al $15 \%$, e ciascuna di questa classificazione è stata suddivisa casualmente in due gruppi di trattamento: $48,4 \%(n=30)$ Gruppo di test utilizzando pelle di tilapia come xenotrapianto e $51,6 \%$ ( $n=32)$ Gruppo di controllo: convenzionale con crema di sulfadiazina. II principale meccanismo di ustione nel gruppo di prova (classificazioni A, B e C utilizzando la pelle di tilapia come trattamento) è stato il $56,25 \%$ di liquidi caldi $(n=18)$. La valutazione dell'intensità del dolore era inferiore nel gruppo di prova rispetto al gruppo di controllo. II numero di medicazioni necessarie durante il trattamento era inferiore nel gruppo di prova, presentando 2,08 $\pm 0,28$ (A), 2,33 \pm 
0,71 (B), 6,10 $\pm 2,02$ (C), mentre nel gruppo di controllo 5,80 $\pm 0,42$ (A), 11,00 $\pm 0,47$ (B), 20,20 $\pm 1,69$ (C) tenendo conto del valore di $P$ come 0,0001 . Per quanto riguarda il numero di giorni per la riepitelizzazione completa, il gruppo di prova ha mostrato $i$ migliori risultati, avendo: 9,77 $\pm 0,83(A), 10,56 \pm 1,13$ (B), 18,10 $\pm 0,99$ (C), mentre il

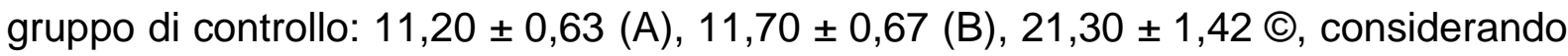
circa $P<0,0147$ (LIMA JUNIOR et al., 2020a)

Un altro studio del Centro per il trattamento delle ustioni a Fortaleza, Ceará, Brasile, ha selezionato 30 bambini, tra i 2 e i 12 anni, ricoverati in ospedale tra maggio 2017 e marzo 2018. Le variabili utilizzate sono state: i giorni totali per la completa guarigione dell'ustione e il numero di medicazioni utilizzate. I pazienti sono stati divisi in due gruppi, in cui il $50 \%$ è stato assegnato al gruppo di test cutaneo tilapia e il resto al gruppo di sulfadiazina d'argento. II numero medio di giorni per completare la riepitelizzazione è stato di 10,47 $\pm 0,74$ nel gruppo della sulfadiazina d'argento e $10,07 \pm 0,46$ nel gruppo cutaneo della tilapia. La differenza tra i due gruppi non era statisticamente significativa $(P=0,0868)$. II numero di medicazioni in anestesia eseguite in pazienti trattati con pelle di tilapia è stato inferiore a quelle eseguite in volontari trattati con sulfadiazina d'argento. Una differenza statisticamente significativa è stata riscontrata anche per il numero di medicazioni senza anestetici. Infine, il numero totale di medicazioni è stato significativamente ridotto nel gruppo cutaneo tilapia $(3,00 \pm 0,76)$ rispetto al gruppo della sulfadiazina d'argento $(9,27 \pm$ 1,39) (LIMA JUNIOR et al., 2019a).

\section{DISCUSSIONE}

Lo studio delle proprietà cutanee della tilapia è un buon punto di partenza per comprenderne il potenziale di utilizzo nelle ustioni. Per questo, vale la pena prima analizzare i risultati di Hu et al. (2017) che ha studiato l'uso di un concentrato di polipeptidi di collagene estratto dalla pelle di tilapia, concentrandosi sul suo potenziale curativo. Saggi in vitro che utilizzano cellule umane preparate hanno dimostrato che l'aggiunta di $50,0 \mu \mathrm{g} / \mathrm{mL}$ del concentrato al tessuto danneggiato ha portato risultati statisticamente significativi in relazione alla velocità di rigenerazione $\mathrm{e}$ 
riepitelizzazione del tessuto. Dall'altro, gli studi in vivo dello stesso autore hanno mostrato risultati promettenti per quanto riguarda la guarigione mediante scottature profonde a spessore parziale nei conigli testati. I risultati sono stati simili a quelli ottenuti con la medicazione allidrogel di collagene, studiata da Ge et al. (2020), per ustioni profonde.

Inoltre, sono stati condotti altri studi per valutare le proprietà della pelle dell'animale. Secondo Alves et al. (2015), la pelle di tilapia presenta fasci di fibre di collagene dense, prevalentemente di tipo 1, con caratteristiche microscopiche simili a quelle della pelle umana, nonché elevata resistenza alla trazione ed estensione, rendendola promettente come biomateriale nella medicina rigenerativa. Lo studio di Ouyang et al. (2018) corrobora questi risultati, così come quelli di Hu et al. (2017), che affronta anche l'attività antibatterica del materiale e ne comprime l'efficienza di polimerizzazione con altre soluzioni disponibili sul mercato, ottenendo un'elevata efficienza, oltre a fornire migrazione e proliferazione cellulare.

Convalidato questo potenziale curativo della pelle di tilapia, è necessario comprendere l'usabilità di questo materiale sotto forma di medicazioni biologiche. Secondo lo studio di Lima Junior (2017), la pelle di tilapia ha una notevole aderenza alle ferite da ustione nei ratti di prova, con risultati positivi durante la guarigione, senza mutazioni significative negli scopi biochimici ed ematologici della funzionalità epatica e renale, costituendo una possibilità positiva come medicazione biologica. $\mathrm{A}$ conferma di questi risultati, lo studio clinico randomizzato di Lima Junior et al. (2020c) ha mostrato una buona aderenza nei pazienti, così come altri vantaggi rispetto ad altre medicazioni, come Aquacel $\mathrm{Ag}{ }^{\circledR}$, che saranno discussi in seguito.

Detto questo, vale la pena confrontare la pelle di tilapia con altre soluzioni esistenti quando si tratta di ustioni. Lima Junior et al. (2020c) e Marcelo e Brandt (2019) hanno promosso studi che non mostrano inferiorità nella pelle di tilapia liofilizzata, rispetto alla sordida medicazione carbossimetilcellulosa impregnata di argento, Aquacel Ag ${ }^{\circledR}$. Lo studio di Lima Junior et al. (2020c) sottolinea che l'uso della pelle di tilapia offre un miglioramento soggettivo della quantità di dolore riportato e del numero di medicazioni richieste, riducendo così il costo del trattamento e il carico di 
lavoro del team. Pertanto, la pelle di tilapia è una buona opzione per il trattamento delle ustioni.

\section{CONCLUSIONE}

Alla luce dei risultati della letteratura riportati nella presente revisione, si conclude che gli studi con la pelle di Tilapia del Nilo, pesci d'acqua dolce, condotti da ricercatori dell'Università Federale del Ceará, che sono stati, dal 2016, i pionieri per brevettare il metodo. Inoltre, è stata una modalità rivoluzionaria con numerosi benefici nel trattamento di pazienti con lesioni cutanee superficiali e profonde che si espandono a diverse aree della medicina, testate in medicina veterinaria, allinterno e all'esterno del paese (LIMA JUNIOR, 2017).

Sulla base dei risultati delle ricerche, i vantaggi dell'utilizzo di pelle di tilapia nel trattamento delle ustioni sono rafforzati, poiché offre una riduzione del dolore del paziente grazie al periodo più lungo aderito alla pelle del paziente. Oltre al ridotto potere di infezione e contaminazione, elevata resistenza dovuta alla grande quantità di collagene presente nella pelle del pesce, con conseguente buona estetica e basso costo, rispetto ad altre forme di trattamento.

Pertanto, i ricercatori sono giunti alla conclusione, rispondendo alla domanda guida, che il nuovo metodo è, sì, un importante progresso nel trattamento delle ustioni, perché la sua occupabilità è confermata, oltre a dimostrare un vantaggio rispetto ad alcune delle principali alternative preesistenti.

\section{RIFERIMENTI}

ALVES, Ana; VERDE, Maria; FERREIRA FILHO, Antônio; SILVA, Paulo; FEITOSA, Victor; LIMA JUNIOR, Edmar; MIRANDA, Marcelo; MORAES FILHO, Manoel. Avaliação microscópica, estudo histoquímico e análise de propriedades tensiométricas da pele de tilápia do Nilo. Revista Brasileira de Queimaduras, v. 14, n. 3, p. 203-210, 2015. 
COSTA, Bruno; LIMA JÚNIOR, Edmar; MORAES FILHO, Manoel; FECHINE, Francisco; MORAES, Maria; SILVA JUNIOR, Francisco; SOARES, Maria; ROCHA, Marina. Use of Tilapia Skin as a Xenograft for Pediatric Burn Treatment: A Case Report. Journal of Burn Care \& Research, v. 40, n. 5, p. 714-717, 2019.

GE, Baosheng; WANG, Haonan; LI, Jie; LIU, Hengheng; YIN, Yonghao; ZHANG, Naili; QIN, Song. Comprehensive Assessment of Nile Tilapia Skin (Oreochromis niloticus) Collagen Hydrogels for Wound Dressings. Marine Drugs, v. 18, n. 4, p. $178,2020$.

HU, Zhang; YANG, Ping; ZHOU, Chunxia; LI, Sidong; HONG, Pengzhi. Marine Collagen Peptides from the Skin of Nile Tilapia (Oreochromis niloticus): Characterization and Wound Healing Evaluation. Marine Drugs, v. 15, n. 4, p. 102, 2017.

LEONTSINIS, Cybele; LIMA JUNIOR, Edmar; MORAIS FILHO, Manoel; BRITO, Maria; ROCHA, Marina; NASCIMENTO, Maria; SILVA JUNIOR, Francisco; MIRANDA, Marcelo. Elaboração de um protocolo para implementação e funcionamento do primeiro banco de pele animal do Brasil: Relato de experiência. Revista Brasileira de Queimaduras, v. 17, n. 1, p. 66-71, 2018.

LIMA JUNIOR, Edmar; PICOLLO, Nelson; MIRANDA, Marcelo; RIBEIRO, Wesley; ALVES, Ana; FERREIRA, Guilherme; PARENTE, Ezequiel, MORAES FILHO, Manoel. Uso da pele de tilápia (Oreochromis niloticus), como curativo biológico oclusivo, no tratamento de queimaduras. Revista Brasileira de Queimaduras, v. 16, n. 1, p. 10-17, 2017.

LIMA JÚNIOR, Edmar. Tecnologias inovadoras: uso da pele da tilápia do Nilo no tratamento de queimaduras e feridas. Revista Brasileira de Queimaduras, v. 16, n. 1, p. 1-2, 2017.

LIMA JÚNIOR, Edmar; MORAES FILHO, Manoel; FORTE, Antônio; COSTA, Bruno; ; FECHINE, Francisco; ALVES, Ana; MORAES, Maria; ROCHA, Marina; SILVA 
JUNIOR, Francisco; SOARES, Maria; BEZERRA, Alane; MARTINS, Camila; MATHOR, Mônica. Pediatric Burn Treatment Using Tilapia Skin as a Xenograft for Superficial Partial-Thickness Wounds: A Pilot Study. Journal of Burn Care \& Research, 2019a.

LIMA JUNIOR, Edmar; MORAES FILHO, Manoel; COSTA, Bruno Almeida; FECHINE, Francisco; MORAES, Maria; SILVA JUNIOR, Francisco; SOARES, MARIA; ROCHA, MARINA, LEONTSINIS, Cybele. Innovative treatment using tilapia skin as a xenograft for partial thickness burns after a gunpowder explosion. Journal of Surgical Case Reports, v. 2019, n. 6, $2019 \mathrm{~b}$.

LIMA JÚNIOR, Edmar; MORAES FILHO, Manoel; COSTA, Bruno; ROHLEDER, Andréa; ROCHA, Marina; FECHINE, Francisco; FORTE, Antonio. ALVES, Ana, SILVA JUNIOR, Francisco; MARINS, Camila; MATHOR, Mônica; MORAES, Maria. Innovative Burn Treatment Using Tilapia Skin as a Xenograft: A Phase II Randomized Controlled Trial. Journal of Burn Care \& Research, v. 41, n. 3, p. 585592, 2020a.

LIMA JUNIOR, Edmar; MORAES, Manoel Odorico; COSTA, Bruno Almeida; UCHÔA, Alex; MARTINS, Camila; MORAES, Maria; ROCHA, Marina; FECHINE, Rodrigo. Treatment of deep second-degree burns on the abdomen, thighs, and genitalia: use of tilapia skin as a xenograft. Revista Brasileira de Cirurgia Plástica (RBCP) - Brazilian Journal of Plastic Sugery, v. 35, n. 2, p. 243-248, 2020b.

LIMA JÚNIOR, Edmar; MORAES FILHO, Manoel ; COSTA, Bruno Almeida; FECHINE, Francisco; ROCHA, Marina; VALE, Mariana; DIÓGENES, Ana; UCHÔA, Alex; SILVA JUNIOR, Francisco; MARTINS, Camila; BANDEIRA, Tereza; RODRIGUES, Felipe; PAIER, Carlos; MORAES, Maria. A Randomized Comparison Study of Lyophilized Nile Tilapia Skin and Silver-Impregnated Sodium Carboxymethylcellulose for the Treatment of Superficial Partial-Thickness Burns. Journal of Burn Care \& Research, v. 42, n. 1, p. 41-48, 2020 c. 
DE MIRANDA, Marcelo José Borges; BRANDT, Carlos Teixeira. Xenoenxerto (pele da Tilápia-do-Nilo) e hidrofibra com prata no tratamento das queimaduras de II grau em adultos. Rev. bras. cir. plást, p. 79-85, 2019.

OUYANG, Qian-Qian; HU, Zhang; LIN, Zhen-Peng; QUAN, Wei-Yan; DENG, YiFeng; LI, Si-Dong; LI, Pu-Wang, CHEN, Yu. Chitosan hydrogel in combination with marine peptides from tilapia for burns healing. International Journal of Biological Macromolecules, v. 112, p. 1191-1198, 2018.

Inviato: Luglio 2021.

Approvato: Dicembre 2021. 